\title{
Chemical Characteristics of Embedded Young Stellar Objects
}

\author{
Michiel R. Hogerheijde \\ Astronomy Department, 601 Campbell Hall, University of California, \\ Berkeley, CA 94720-3411, USA
}

\begin{abstract}
Based on theoretical considerations, the chemistry around embedded young stellar objects is expected to be governed by the interplay between gas-phase reactions, condensation of molecules onto dust grains, grain surface reactions, and evaporation of altered ice mantles near the star and in the outflow. I discuss the chemical characteristics of embedded young stellar objects, with special emphasis on these processes.
\end{abstract}

\section{Introduction}

The contributions in this volume focus on the chemical evolution from interstellar clouds through the process of star formation leading up to the origins of planetary systems. Here I will discuss the earliest stages of this evolution: the chemical characteristics of the collapsing cloud cores around young stars. Ohashi (this volume) discusses the physical structure of these collapsing envelopes. The chemistry during the stage preceding the collapse phase, dominated by freezing out of molecules from the gas phase onto dust grains, is covered by Rawlings and by Caselli (this volume). I limit the discussion to low-mass stars $\left(<2-3 \mathrm{M}_{\odot}\right)$. The physics and chemistry associated with the formation of stars of larger mass is different in many aspects, as discussed by van Dishoeck and by Macdonald (this volume).

Why are we interested in the chemical characteristics of embedded young stellar objects (YSOs) ? First, we want to understand how dark cloud chemistry evolves through cloud collapse, determining the composition of the solar nebula. Second, we want to identify chemical species which are robust tracers of star formation, or which specifically trace certain regions or processes. And finally, we want to evaluate the influence of outflows driven by many YSOs as they shock surrounding material and destroy dust grains and ice mantles. This contribution is mostly limited to the first two topics; Garay (this volume) discusses outflows in more detail.

Section 2 briefly summarizes chemical models of star formation, highlighting specific processes that YSOs may exhibit. Section 3 takes inventory of the chemical characteristics of a number of well studied YSOs, followed by results of recent surveys of (limited) samples of YSOs in section 4. Section 5 summarizes the main conclusions and sketches directions for future work. Recent reviews of the material presented here include van Dishoeck \& Blake (1998), van Dishoeck \& Hogerheijde (1999), and Langer et al. (2000). 


\section{Models of Chemistry in Star-Forming Regions}

This section gives a brief overview of the chemistry expected in YSO envelopes; more detailed accounts can be found in the contributions by Bergin and by Herbst (this volume). Many chemical models take the predominantly atomic composition of diffuse clouds as initial conditions, and follow the chemistry through the formation of a dense core. Four processes determine the ensuing chemistry: gas-phase reactions, condensation of molecules in ice mantles on dust grains, grain-surface reactions, and release of ices when the grains are heated. For the typical conditions in dark clouds, the time scales of cloud core formation, the creation of a dark-cloud chemistry, and the condensation of a significant fraction of the molecules from the gas phase onto grains all take place on very similar time scales of a few times $10^{5}$ to $10^{6} \mathrm{yr}$. All these processes should therefore be considered simultaneously.

Gas-phase chemistry is dominated by ion-molecule reactions, which are much more efficient than reactions between neutrals in the dilute environment of interstellar clouds. Many reaction schemes involving the main elements (other than hydrogen) $\mathrm{C}, \mathrm{O}, \mathrm{N}$, and $\mathrm{S}$ therefore start with reactions with $\mathrm{H}_{3}^{+}$. This molecular ion is formed after cosmic-ray ionization, at a rate $\zeta_{\mathrm{CR}}=5 \times 10^{-17}$ $\mathrm{s}^{-1}$ (Williams et al. 1998), of the main molecular species $\mathrm{H}_{2}$ to $\mathrm{H}_{2}^{+}$, followed by $\mathrm{H}_{2}^{+}+\mathrm{H}_{2} \rightarrow \mathrm{H}_{3}^{+}+\mathrm{H}$. In the developing chemistry, roughly two groups of species can be distinguished: 'early-time' and 'late-time' species. Early-time species are molecules whose formation requires the presence of $\mathrm{C}^{+}$or $\mathrm{C}$. As a result of the gradual transition of carbon from $\mathrm{C}^{+}$to $\mathrm{C}$ and $\mathrm{CO}$, these species first increase in abundance and then decrease toward their equilibrium value. Examples are CS, $\mathrm{CCS}, \mathrm{CN}, \mathrm{HCN}$, and long carbon chains. Late-time species have abundances rising steadily throughout the evolution. They include molecules whose formation is either independent of carbon, or whose main destruction pathways involves $\mathrm{C}$ or $\mathrm{C}^{+}$. Examples of late-time species are $\mathrm{N}_{2}, \mathrm{NH}_{3}, \mathrm{~N}_{2} \mathrm{H}^{+}, \mathrm{SO}$, and $\mathrm{HCO}^{+}$.

While the gas-phase chemistry is evolving, molecules are freezing out in the cold and dense regions of the cloud. In the ice mantles which are formed in this way, much longer time scales are available for reactions, allowing hydrogenation and oxidation to take place. These surface reactions are thought to be the main sources of $\mathrm{H}_{2} \mathrm{CO}, \mathrm{CO}_{2}$, and $\mathrm{CH}_{3} \mathrm{OH}$ (Tielens \& Charnley 1997). The review by Herbst (this volume) treats the many uncertainties involved in models of grain-surface chemistry in detail. The species formed through grainsurface reactions are selectively released back into the gas phase when the grains are heated above their sublimation temperature. Grain particles are heated by direct radiation from the star, through slow shocks which do not destroy the grains or the molecules, and through spot-heating due to cosmic-ray hits or exothermic surface reactions. Especially the latter process could be responsible for a continuous feedback of grain-surface products into the gas phase. Grain destruction leads to the release of $\mathrm{Si}$, which rapidly reacts to $\mathrm{SiO}$ (Schilke et al. 1997), providing a tell-tale sign of shocked gas.

The picture sketched here is a very simplistic one of the chemistry of dark cloud cores and YSO envelopes. Major uncertainties are whether the initial conditions are fully atomic or partially molecular, the length of time which a cloud core spends in a dense phase before collapsing and forming a star, and the interaction of gas-phase and grain-surface chemistry. In spite of these uncertainties, 
the expected trend is chemical differentiation between early-time and late-time species, the depletion of $\mathrm{CO}$ and other species in the densest and coldest regions, and the return of processed ices near the star and along the outflow.

\section{Inventory of Chemical Characteristics}

Chemical differentiation is perhaps most clearly seen toward the starless core L1498 (Kuiper, Langer, \& Velusamy 1996; Willacy, Langer, \& Velusamy 1998). $\mathrm{CO}$ is strongly depleted at the center of this core, which is traced itself by far-infrared dust continuum and $\mathrm{NH}_{3}$. The early-time species CS and CCS trace a shell around the core. A similar behavior of CCS is seen toward the deeply embedded YSOs B 335 by Velusamy, Kuiper, \& Langer (1995). However, toward many embedded YSOs this nice picture is significantly confused because of heating by the outflow and the central star.

A good example is the $30^{\prime \prime}$ binary NGC 1333 IRAS $4 \mathrm{~A}$ and $4 \mathrm{~B}$. Blake et al. (1995) carried out a line survey of both sources in the $230 \mathrm{GHz}$ and $345 \mathrm{GHz}$ atmospheric windows, and find that most molecules have typical dark-cloud abundances with respect to $\mathrm{CO}$, but that all species are depleted by factors of 10-20 with respect to the dust continuum emission. The spectra are characterized by narrow peaks of $1-2 \mathrm{~km} \mathrm{~s}^{-1}$ and broad line wings extending to $20 \mathrm{~km} \mathrm{~s}^{-1}$ from line center, associated with the outflows driven by the objects. Although spatially coincident, the emission from the dense envelopes and from material affected by the outflows is separate in velocity space, allowing abundance determinations of both. The material responsible for the line wings is enhanced in species thought to originate in grain-surface reactions, like $\mathrm{H}_{2} \mathrm{CO}, \mathrm{CH}_{3} \mathrm{OH}$, and SO. Recent interferometric observations by Di Francesco et al. (1999) also reveal the spatial separation between $\mathrm{N}_{2} \mathrm{H}^{+}$tracing the dense envelopes and $\mathrm{H}_{2} \mathrm{CO}$ outlining the highly-collimated outflows.

Another recent observation toward NGC 1333 IRAS 4A of particular interest is the first detection of interstellar $\mathrm{H}_{2} \mathrm{D}^{+}$, deuterated $\mathrm{H}_{3}^{+}$, by Stark, van der Tak, \& van Dishoeck (1999). $\mathrm{H}_{3}^{+}$itself, pivotal in interstellar chemistry (§2), has been detected only recently in the interstellar medium (Geballe \& Oka 1997; McCall et al. 1999). The detection of the $1_{10}-1_{11}$ ground-state transition of ortho- $\mathrm{H}_{2} \mathrm{D}^{+}$at $372 \mathrm{GHz}$ toward IRAS $4 \mathrm{~A}$ is an excellent illustration of how detailed physical models are required to infer abundance information. Although the excitation of this line requires temperatures of around $100 \mathrm{~K}$, a temperature only reached in a very small fraction of the material in the cold envelope, the selectivity at low temperatures of the $\mathrm{H}_{3}^{+}+\mathrm{HD} \rightleftharpoons \mathrm{H}_{2} \mathrm{D}^{+}+\mathrm{H}_{2}$ reaction toward the formation of $\mathrm{H}_{2} \mathrm{D}^{+}$is sufficient to yield an observable column density of the deuterated species in the cold material. Using a detailed description for the density and temperature in this source, based on previous molecular line observations, the authors show that a simple gas-phase low-temperature chemistry model can explain the observed line intensity. They infer a beam-averaged $\mathrm{H}_{2} \mathrm{D}^{+}$abundance of $3 \times 10^{-12}$.

Are depletion factors of more than an order of magnitude common in the dense envelopes around deeply embedded YSOs? A study by Hogerheijde et al. (1999) of the deeply embedded YSO Serpens SMM 1 suggests that such high depletion factors are either short lived or dependent on the star-forming environ- 


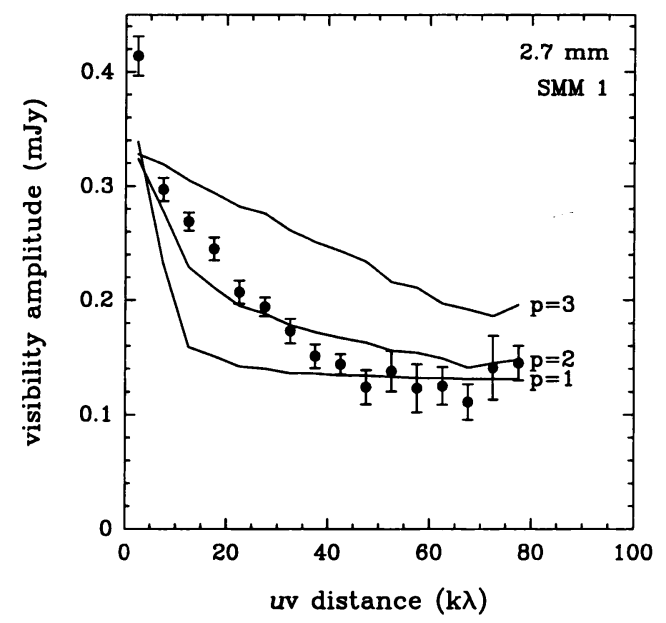

Figure 1. Continuum flux at $2.7 \mathrm{~mm}$ observed toward the deeply embedded YSO Serpens SMM 1 with the Owens Valley Millimeter Array (points) as function of projected baseline length. The emission is resolved and well fit by a radial power law of index $-2.0 \pm 0.5$ (solid lines) (from: Hogerheijde et al. 1999).

ment. The Serpens molecular cloud is forming a dense cluster (Eiroa \& Casali 1992; Casali, Eiroa, \& Duncan 1993), and SMM 1, together with half a dozen other embedded objects, represents the most recent phase of star-forming activity (Testi \& Sargent 1998). Using the Owens Valley Millimeter Array, Hogerheijde et al. resolve the continuum emission from the $9 \mathrm{M}_{\odot}$ envelope around SMM 1 (Fig. 1). The data indicate a density distribution following a radial power law with index $-2.0 \pm 0.5$, consistent with models for protostellar collapse (e.g. Shu 1977). Using this density distribution and a self-consistently calculated temperature profile for the source's bolometric luminosity of $77 \mathrm{~L}_{\odot}$, molecular abundances and a $\mathrm{CO}$ depletion of a factor of 3-10 are derived. However, the single-dish spectra (Fig. 2) of all but the most optically thin species are dominated by the outflow wings or by emission from $\sim 10 \%$ of the material at an elevated temperature of $\sim 100 \mathrm{~K}$. Especially the ${ }^{13} \mathrm{CO} J=6-5$ lines and several highly-excited $\mathrm{H}_{2} \mathrm{CO}$ lines indicate the presence of this warm material, which may be associated with heating by the outflow or by stellar radiation. The selfconsistent temperature modeling indicates that the temperature reaches $300 \mathrm{~K}$ in the inner few hundred AU, but the exact column density depends critically on the geometry of the envelope on these unresolved scales.

On the small scales $(\sim 1000 \mathrm{AU})$ probed by the interferometer (Fig. 3), optically thin species like $\mathrm{C}^{18} \mathrm{O}$ and $\mathrm{H}^{13} \mathrm{CO}^{+}$appear to trace only a small fraction of the envelope mass. But after taking into account the spatial filtering by the interferometer, their line intensities are consistent with the envelope model. This illustrates that any investigation of abundances on small scales requires that, in addition to a fiducial model for the physical structure of the envelope on small scales, the spatial filtering of the interferometer is explicitly taken into account or that zero-spacing information is included (see also van Dishoeck \& 

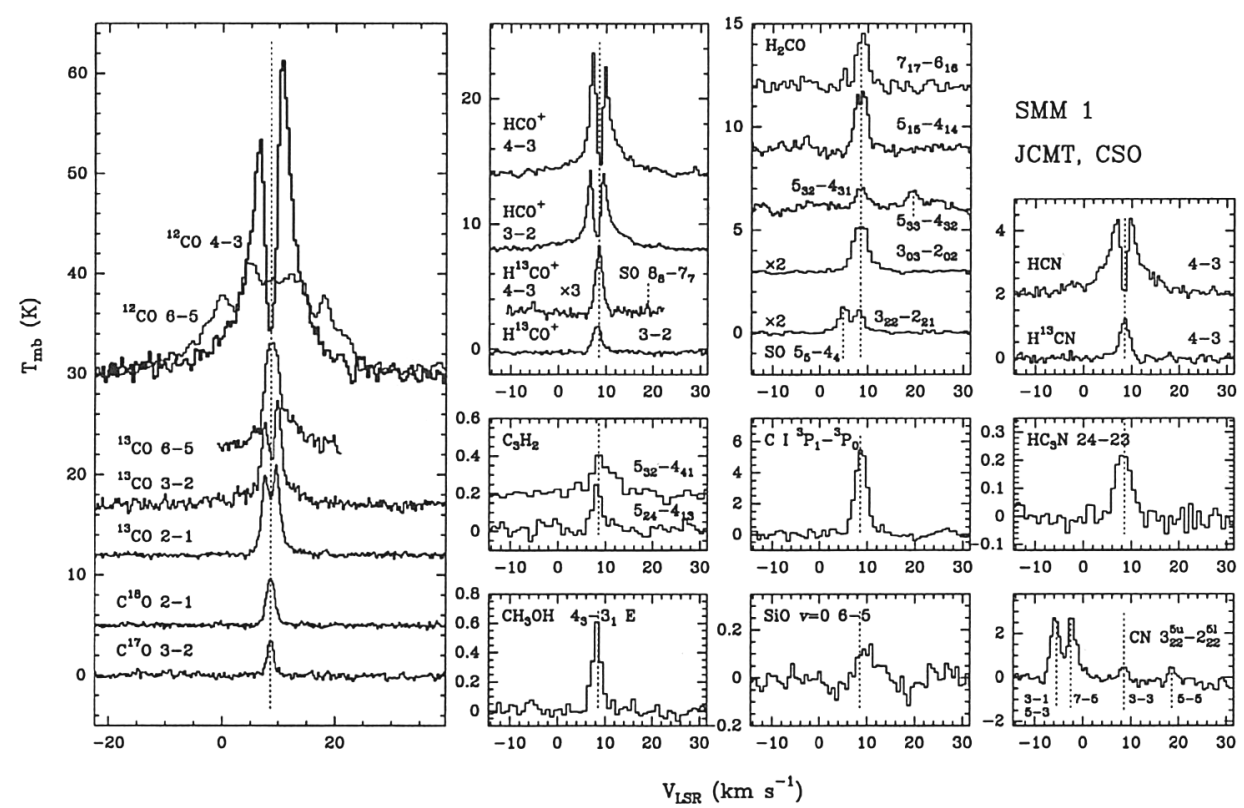

Figure 2. Overview of spectra observed with the James Clerk Maxwell Telescope and the Caltech Submillimeter Observatory toward Serpens SMM 1. Together with the physical model derived from the continuum observations presented in Fig. 1, these spectra yield abundance estimates. Many lines, however, are dominated by material in the outflow or by a small fraction of the gas that is at temperatures exceeding $100 \mathrm{~K}$ (from: Hogerheijde et al. 1999).

Hogerheijde 1999 for a detailed discussion on the the steps involved in abundance determinations). In the main isotope lines of $\mathrm{HCO}^{+}, \mathrm{HCN}, \mathrm{SO}$, and $\mathrm{SiO}$ (Fig. 3 ), the observations reveal the interaction of the outflow with the surrounding material. $\mathrm{HCO}^{+}$and $\mathrm{HCN}$ appear to line the northwest outflow cavity, while $\mathrm{SiO}$ and $\mathrm{SO}$ emission along the axis of the southeast flow probably traces shocks and grain destruction.

The best studied embedded YSO is likely IRAS 16293-2422 (e.g. Blake et al. 1994; van Dishoeck et al. 1995). A 5" (800 AU) separation binary (Mundy et al. 1992), this source drives two bipolar outflows which leave their imprint on the chemistry. From single-dish observations, Blake et al. (1994) and van Dishoeck et al. (1995) deduce the following structure. A central region of 500-1500 AU is dense $\left(2 \times 10^{7} \mathrm{~cm}^{-3}\right)$ and warm $(80 \mathrm{~K})$, and rich in $\mathrm{SO}, \mathrm{SiO}, \mathrm{CH}_{3} \mathrm{OH}, \mathrm{SO}_{2}$, and $\mathrm{H}_{2} \mathrm{CO}$. This region is likely heated by the stellar radiation or shocked by the outflows. The interaction of the outflows with the surrounding material is traced by recent interferometer results by Mundy et al. (1999). The evaporation of ice mantles is also traced by detection of water emission in observations with the Infrared Space Observatory (Ceccarelli et al. 1998b) at an inferred abundance of $2 \times 10^{-5}$, and of $\mathrm{D}_{2} \mathrm{CO}$ (Ceccarrelli et al. 1998a). A deuteration fraction of $\leq 0.1$ is inferred, and this high value is interpreted as evidence for formation at low- 


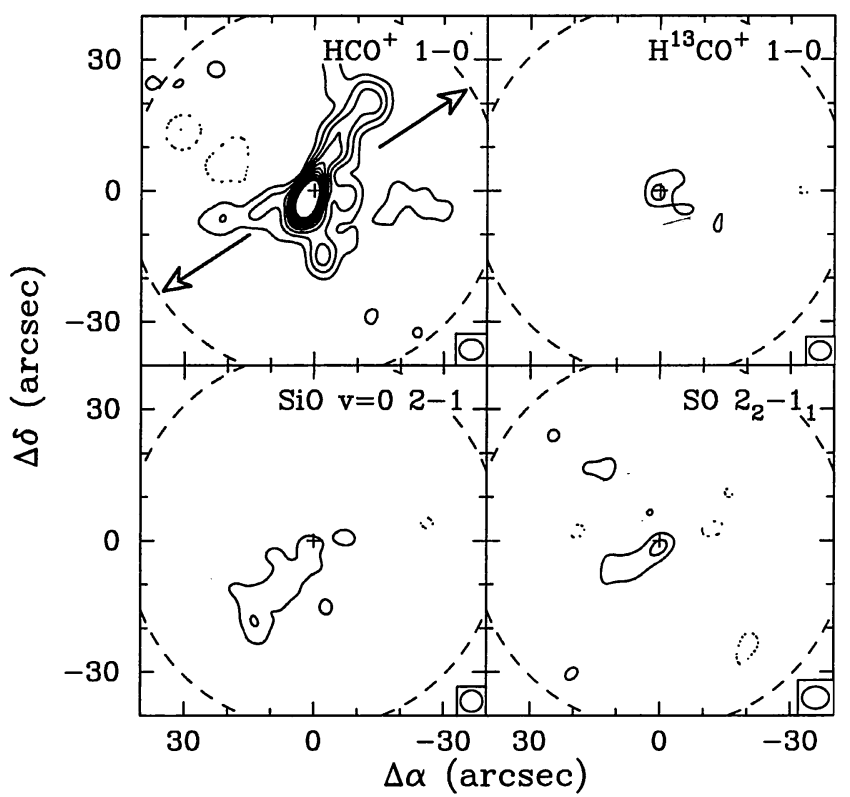

Figure 3. Images of $\mathrm{HCO}^{+}, \mathrm{H}^{13} \mathrm{CO}^{+}, \mathrm{SiO}$, and $\mathrm{SO}$ emission of Serpens SMM 1 observed with the Owens Valley Millimeter Array. The $\mathrm{H}^{13} \mathrm{CO}^{+}$emission traces dense gas in the inner envelope; $\mathrm{HCO}^{+}$outlines the outflow, indicated by the arrows; $\mathrm{SiO}$ and $\mathrm{SO}$ lie along the southeast extension of the flow and probably trace grain destruction or ice mantle evaporation.

temperatures on grain surfaces and subsequent evaporation. Surrounding this central region is a $2000 \mathrm{AU}$ circumbinary envelope of $40 \mathrm{~K}$ and $10^{6}-10^{7} \mathrm{~cm}^{-3}$, traced in $\mathrm{HCO}^{+}, \mathrm{CS}$, and $\mathrm{H}_{2} \mathrm{CO}$. On larger scales, the extended cold $(10-20 \mathrm{~K})$ and low-density $\left(3 \times 10^{4}-2 \times 10^{5} \mathrm{~cm}^{-3}\right)$ core is probed in early-time species like $\mathrm{CN}, \mathrm{C}_{3} \mathrm{H}$, and $\mathrm{C}_{3} \mathrm{H}_{2}$, as well as through self-absorption features in the $\mathrm{HCO}^{+}$ and HCN spectra.

\section{Surveys of YSOs}

Recent years have seen the first results of several surveys of embedded YSOs, addressing the physical and chemical structure of their envelopes (Hogerheijde et al. 1997, 1998, illustrated in Fig. 4; Ohashi et al. 1996). The main focus of this work was the clarification of the physical structure of the YSO envelopes, required for any reliable abundance determination (Ohashi, this volume). Of particular interest in this respect is the identification of $\mathrm{HCO}^{+}$and $\mathrm{N}_{2} \mathrm{H}^{+}$as robust tracers of the circumstellar envelopes (Hogerheijde et al. 1997; Benson, Caselli, \& Myers 1998). Both species are formed along similar paths through reactions of the main carbon, respectively, nitrogen bearing molecules $\mathrm{CO}$ and $\mathrm{N}_{2}$ with $\mathrm{H}_{3}^{+}$. The destruction path for both species is through reactions with $\mathrm{H}_{2} \mathrm{O}$, or, if water is depleted in gas below $100 \mathrm{~K}$, through $\mathrm{N}_{2} \mathrm{H}^{+}+\mathrm{CO} \rightarrow \mathrm{HCO}^{+}+\mathrm{N}_{2}$ 


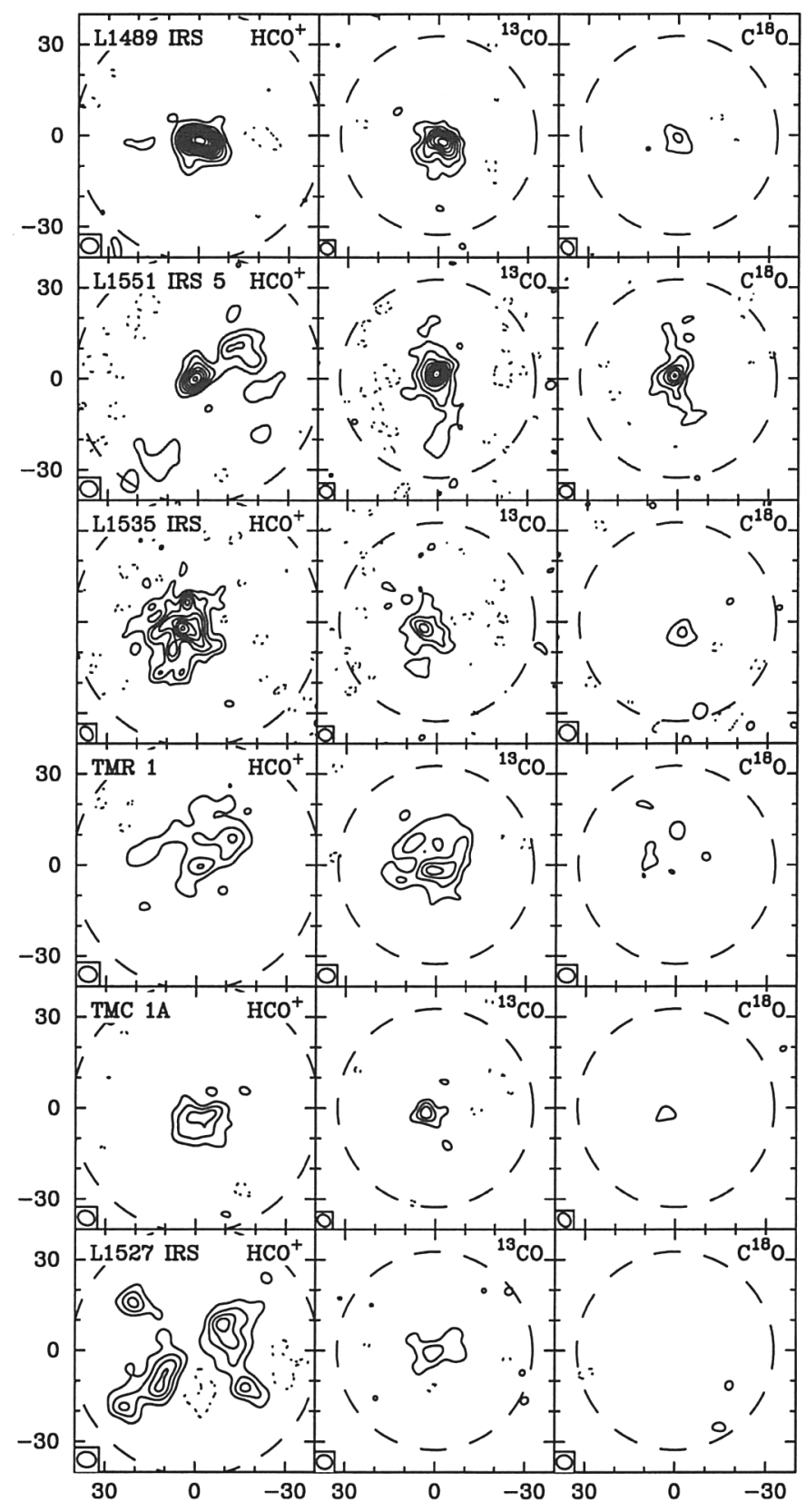

Figure 4. Images of $\mathrm{HCO}^{+} J=1-0,{ }^{13} \mathrm{CO} 1-0$, and $\mathrm{C}^{18} \mathrm{O} 1-0$ obtained with the Owens Valley Millimeter Array toward six embedded YSOs from the sample of Hogerheijde et al. $(1997,1998)$. 
and $\mathrm{HCO}^{+}+e^{-} \rightarrow \mathrm{CO}+\mathrm{H}$. Thus, the abundances of $\mathrm{HCO}^{+}$and $\mathrm{N}_{2} \mathrm{H}^{+}$remain high throughout the envelope even where $\mathrm{CO}$ and $\mathrm{N}_{2}$ freeze out, because their destruction pathways are suppressed as well. In gas above the sublimation temperature of $\mathrm{CO}(20 \mathrm{~K})$ but below that of $\mathrm{H}_{2} \mathrm{O}(100 \mathrm{~K}), \mathrm{HCO}^{+}$remains present while $\mathrm{N}_{2} \mathrm{H}^{+}$disappears; in gas above $100 \mathrm{~K}$ both molecules are absent (G. Blake, priv. comm.). Observations indeed reflect this expected behaviour.

\section{Conclusion}

The last few years have seen great progress in the characterization of the chemistry around embedded YSOs, thanks to the increased sensitivity of millimeter arrays at Owens Valley, Hat Creek, Plateau de Bure, and Nobeyama. These instruments enabled the construction of a consistent physical picture for the protostellar environment by revealing the relevant scales of a few hundred to a few thousand AU. Together with single-dish results from the James Clerk Maxwell Telescope, the Caltech Submillimeter Observatory, and the IRAM 30m telescope, the picture that emerges matches theoretical expectations of chemical differentiation between early-time and late-time species, depletion, grain-surface reactions, and ice-mantle evaporation.

Of particular interest are the identification of robust tracers of the dense envelopes like $\mathrm{HCO}^{+}$and $\mathrm{N}_{2} \mathrm{H}^{+}$. The low-temperature phase of the chemical evolution, which depletions may render hard to observe in emission lines, are rendered observable through high amounts of deuteration in the gas phase $\left(\mathrm{H}_{2} \mathrm{D}^{+}\right)$ and on grain surfaces followed by evaporation at a later stage $\left(\mathrm{D}_{2} \mathrm{CO}\right)$. A similar thermal 'memory' may be present in the ortho-to-para ratio of $\mathrm{H}_{2} \mathrm{CO}$ (Dickens \& Irvine 1999).

The prospects for this field in the coming years are exciting. With the consolidation of a consistent physical picture for embedded YSOs, coupled with detailed radiative transfer calculations (see Black, this volume), reliable abundance estimates can be derived. Or even better, using chemical models as an input, the comparison between calculated and observed spectra can directly test our understanding of the chemistry. The construction of the Smithsonian Millimeter Array and the Atacama Large Millimeter Array will result in a wealth of new observations. It is therefore likely that in the next few years we will go beyond the chemical characteristics of embedded YSOs and will start to probe their chemical structure.

Acknowledgments. MRH gratefully acknowledges the IAU and the organizers of the symposium for financial assistance. MRH's research is supported by the Miller Institute for Basic Research in Science.

\section{References}

Benson, P.J., Caselli, P., \& Myers, P.C. 1998, ApJ, 506, 743

Blake, G.A., Sandell, G., van Dishoeck, E.F., Groesbeck, T.D., Mundy, L.G., \& Aspin, C. 1995, ApJ, 441, 689

Blake, G.A., van Dishoeck, E.F., Jansen, D.J., Groesbeck, T.D., \& Mundy, L.G. 1994, ApJ, 428, 680 
Casali, M.M., Eiroa, C., \& Duncan, W.D. 1993, A\&A, 275, 195

Ceccarelli, C., Castets, A., Loinard, L., Caux, E., \& Tielens, A.G.G.M. 1998a, A\&A, 338, L43

Ceccarelli, C., Caux, E., White, G.J., Molinari, S., Furniss, I., Liseau, R., Nisini, B., Saraceno, P., Spinoglio, L., \& Wolfire, M. 1998b, A\&A, 331, 372

Di Francesco, J., Myers, P.C., Wilner, D., Ohashi, N., \& Mardones, D. 1999, in preparation

Dickens, J.E. \& Irvine, W.M. 1999, ApJ, 518, 733

Eiroa, C. \& Casali, M.M. 1992, A\&A, 262, 468

Geballe, T.R. \& Oka, T. 1996, Nature, 384, 334

Hogerheijde, M.R., van Dishoeck, E.F., Blake, G.A., \& van Langevelde, H.J. 1997, ApJ, 489, 293 1998, ApJ, 502, 315

Hogerheijde, M.R., van Dishoeck, E.F., Salverda, J.M., \& Blake, G.A. 1999, ApJ, 513, 350

Kuiper, T.B.H., Langer, W.D., \& Velusamy, T. 1996, ApJ, 468, 761

Langer, W.D., van Dishoeck, E.F., Bergin, E.A., Blake, G.A., Tielens, A.G.G.M., Velusamy, T., \& Whittet, D. 2000, in Protostars and Planets IV, eds. V.G. Mannings, A.P. Boss, \& S.S. Russell (Tucson: University of Arizona Press), in press

McCall, B.J., Geballe, T.R., Hinkle, K.H., \& Oka, T. 1999, ApJ, 522, 338

Mundy, L.G., Wootten, A., Wilking, B.A., Blake, G.A., \& Sargent, A.I. 1992, ApJ, 385, 306

Mundy, L.G., et al. 1999, in preparation

Ohashi, N., Hayashi, M., Kawabe, R., \& Ishiguro, M. 1996, ApJ, 466, 317

Schilke, P., Walmsley, C., Pineau des Forêts, G., \& Flower, D. 1997, A\&A, 321, 293

Shu, F.H. 1977, ApJ, 214, 488

Stark, R., van der Tak, F. F.S., \& van Dishoeck, E.F. 1999, ApJ, 521, L67

Testi, L. \& Sargent, A.I. 1998, ApJ, 508, L91

Tielens, A.G.G.M. \& Charnley, S.B. 1997, in Planetary and Interstellar Processes Relevant to the Origins of Life, ed. D.C.B. Whittet (Dordrecht: Kluwer Academic Publishers), 23

van Dishoeck, E.F. \& Blake, G.A. 1998, ARA\&A, 36, 317

van Dishoeck, E.F., Blake, G.A., Jansen, D.J., \& Groesbeck, T.D. 1995, ApJ, 447,760

van Dishoeck, E.F. \& Hogerheijde, M.R. 1999, in The Origin of Stars and Planetary Systems, eds. C.J. Lada \& N.D. Kylafis (Dordrecht: Kluwer Academic Publishers), 97

Velusamy, T., Kuiper, T. B.H., \& Langer, W.D. 1995, ApJ, 451, L75

Willacy, K., Langer, W.D., \& Velusamy, T. 1998, ApJ, 507, L171

Williams, J.P., Bergin, E.A., Caselli, P., Myers, P.C., \& Plume, R. 1998, ApJ, 503,689 


\section{Discussion}

M. Guelin: The $\lambda \lesssim 1 \mathrm{~mm}$ thermal dust emission in very cold cores varies as $\mathrm{T}_{d}^{\beta}$ with $\beta>1$. The molecular line emission (e.g. $\mathrm{C}^{18} \mathrm{O} \mathrm{J}=1-0$ ) as $\mathrm{T}_{k}^{-1}$ when the lines are optically thin. Finally, the emission from highly polar molecules like $\mathrm{N}_{2} \mathrm{H}^{+}$or $\mathrm{HCN}$ is boosted toward dense cores. The derivation of the CO depletion factor from a comparison of the dust, $\mathrm{CO}$ and $\mathrm{N}_{2} \mathrm{H}^{+}$emissions is therefore not straightforward. Could you comment on this?

$M$. R. Hogerheijde: I totally agree with this comment; it in fact underlines one of the main points of my talk, i.e., detailed physical models and correct treatment of the radiative transfer and molecular excitation are required before fiducial statements can be made about the chemical structure of YSO envelopes. With the increasing knowledge of the physical structure of these cold cores, I hope that more robust, quantitative statements about $\mathrm{CO}$ depletion etc. are now possible.

S. Takano: I was impressed by the high $(\sim 10 \%)$ deuterium concentration of formaldehyde $\left(\left[\mathrm{D}_{2} \mathrm{CO}\right] /\left[\mathrm{H}_{2} \mathrm{CO}\right]\right)$. But how reliable is the value of concentration? The lines of normal species can easily be optically thick.

M. R. Hogerheijde: The authors (Ceccarelli et al. 1998, A\&A, 338, L43) show that even the $\mathrm{D}_{2} \mathrm{CO}$ line they observed is self-absorbed. Their analysis yields an upper limit to the $\mathrm{D}_{2} \mathrm{CO} / \mathrm{H}_{2} \mathrm{CO}$ ratio of $\leq 0.1$. If the lines are only moderately optically thick, the $\mathrm{D}_{2} \mathrm{CO} / \mathrm{H}_{2} \mathrm{CO}$ is not much smaller than $10 \%$. Isotopic-C observations $\left(\mathrm{H}_{2}^{13} \mathrm{CO}\right.$, etc.) should confirm this. 